\title{
Pattern Primitive Library Construction and Feature Analysis of Kirgiz Textile Pattern
}

\author{
Juan Qian", 2, ", Aimin Xiao ${ }^{1,2}$, Xiaoyu Xin ${ }^{1,2}$, Mingjie He ${ }^{1}$ \\ ${ }^{1}$ Institute of Textile and Clothing, Xinjiang University, Urumqi, China \\ ${ }^{2}$ Silk Road Apparel Research and Development Center, Xinjiang University, Urumqi, China \\ Email address: \\ qianjuan0829@163.com (Juan Qian),495178065@qq.com (Aimin Xiao),479790302@qq.com (Xiaoyu Xin), \\ 1730487461@qq.com (Minjie He) \\ ${ }^{*}$ Corresponding author
}

\section{To cite this article:}

Juan Qian, Aimin Xiao, Xiaoyu Xin, Mingjie He. Pattern Primitive Library Construction and Feature Analysis of Kirgiz Textile Pattern. Humanities and Social Sciences. Vol. 7, No. 6, 2019, pp. 185-190. doi: 10.11648/j.hss.20190706.11

Received: November 4, 2019; Accepted: November 22, 2019; Published: December 2, 2019

\begin{abstract}
Textile patterns in each minority which contain cultural, historical, artistical, and reginal features are precious because of its unique aesthetic characteristics. More than 78 typical classical patterns of Kirgiz Yormac which is a kind of Kirgiz home-made textile are collected through field investigation, and digitalized by CorelDraw based on the concept of pattern primitives. Three methods for obtaining the pattern primitive units are proposed, and a pattern primitive library is established. It is found that the most popular Yormac pattern items are the ram horn, the flying eagles, and geometric figures, with a symmetrical configuration and structure. Red is the most epidemic color with the proportion over $70 \%$ of the pattern collected. By statistically and literature analysis, it can be concluded that Kirgiz textile pattern comes from life, being used in life. The custom, implication, spirit of Kirgiz shown in pattern come down in one continuous line with national pattern, and are highly in conformity with the culture and tradition of Chinese nation. The feature analysis of Yormac pattern, and construction of pattern primitive library is done for better comprehension of Kirgiz culture, and thus protecting and inheriting the Kirgiz intangible culture heritage.
\end{abstract}

Keywords: Kirgiz, Yormac, Pattern, Pattern Primitive Library

\section{Introduction}

The pattern in Home-textiles of every minority which contain cultural characteristics and ethnic aesthetic tastes are a precious part of the Chinese traditional culture and art because of its historical, regional, social and cultural features, as well as a special way to express the beauty of the word and life for some people especially who lives a relatively traditional way in history and nowadays[1]. Kirgiz tribe whose people lives a nomadic life in history mainly settled in Tianshan Mountains and Pamir plateau regions, is one of minorities in northern western China[2]. Under the influence of Chinese central plains culture and Xinjiang minority ethnic culture, a unique aesthetic style in Kirgiz textile decorative arts has formed which is different compared with other Chinese tribe, and worth for further discussion.

The Yormac studied in this paper is necessities of Kirgiz daily life which emerged and developed in the course of their long history of migration. The material of Yormac which is available from gazed animals, such as sheep, alpaca, was collected, carefully selected and dyed. After carding, yarning and twisting by hand, various strip fabrics within a width from 5 to $30 \mathrm{~cm}$ woven on a horizontal wooden loom are called Yormac, which are commonly utilized for binding, fixing, wearing, decorating and storage in their routine life[3]. The fabricating process which is carried on by Kirgiz housewives is similar despite there are quite a few kinds of Yormac products with different color and pattern. With moving the wooden warp separator back and forth, beating-up the stick on a three-point ground loom, a series of plain and jacquard fabrics are woven by changing the color of yarn, direction of twist, the number and position of thread selected [4]. These Yormac products have various Kirgiz names and patterns due to different application and width. For example, Kalatirim which is a kind of narrower belt with the width between $5 \mathrm{~cm}$ 
to $12 \mathrm{~cm}$ is generally used for bunding to reinforce their yurt. Qiepukan which is made of raw alpaca fiber is stitched the strip fabric together to sew a coat for Kirgiz men as a symbol of wealth. Kaqiare and Parazi with the width between $10 \mathrm{~cm}$ to $30 \mathrm{~cm}$ are sewing into a plain cloth for decorating the living room. And also, An Ayakekapu is sewn for storage and Krujun for their horses [4, 5].

The present study on ethnic textile pattern in China is paid more attention on cultural, artistic, ethnical, and historical features in southern China than north since the end of the 20th century. Researchers have done a lot of work with fruitful results, especially in ethnic pattern of home-made textiles [6-9]. There are many kinds of homemade textiles with decoration to cater for the Kirgiz nomadic lifestyle. Embroidery, felting, weaving and knitting are the primary ways to ornament which are recorded and analyzed in a cultural and ethnical perspective, such as the work done by Prf. $W$. Хиеуи [2, 10-13]. These research above which was focused on decorative method rather than the pattern itself do enrich the Kirgiz national handicraft culture, and promoted to protect Kirgiz traditional intangible cultural heritage, but not enough. As a part of intangible culture heritage, Kirgiz decorative pattern has its own distinctive artistic features and unique form, and contain profound and diversified cultural connotation. From the point of cultural and artistic view, it is a unique artistic style combined with religious, totem, regional, nomadic and other cultural significations $[1,14,15]$. However, cultural analysis cannot be the best way to reserve all of the information of an ethnic pattern in digital age nowadays.

with the advancing of digital technology, the digital regeneration project of Peking Opera facial mask and paper-cut in China have both innovated the methods and forms in studying the art of textile pattern [16], greatly accelerating the development of digital technology in the traditional art of pattern simultaneously. In order to provide better protection and inheritance of the Xinjiang folk craft pattern and improve the ethnic pattern's differentiation degrees of national characteristic, computer aided design technology was used by Zhao Hai-ying team who came from Xinjiang Normal University, and the concept of basic unit of patterns are proposed in 2011 to retain basic national characteristics of the pattern by establishing multi-level pattern primitive library[17-19]. At 2017, Wu Yun team proposed a digital construction method based on database for basic ethnic pattern [20]. These work all introduce a good idea for better inheritance and innovation the Kirgiz Yormac pattern.

From the historical and cultural perspective, Kirgiz pattern was concerned, but the digital inheritance and the methods of innovation were paid less attention to. Therefore, combined with the concept of pattern unit, the fabric of Yormac which was homemade by Kirgiz people is chosen to research here.
The most typical patterns were segmented into basic units, and then a pattern primitive library was established so as to a novel pattern which is still with Kirgiz style was generated subsequently.

\section{Method}

\subsection{Materials and Tool}

\subsubsection{Yormac}

All of the Yormac pattern were collected through field investigation in order to keep the pattern in an original state. There are two autonomous prefectures where the Kirgiz reside in Xinjiang were visited, and the different types of Yormac was pictured and collected in three districts of the two as more as we can. The older Yormac which is gathered from Kirgiz herdsman is the better than market, because the pattern is more delicate and classical.

\subsubsection{Computer-Aided Design (CAD)}

CAD technology contributes to designing digital patterns, mostly applying them in modern processing technology. There are obvious advantages in keeping a pattern in original state, and provide great convenience for designers in saving, modifying, and redesigning a new pattern immediately. So how to use computer graphics technology to digitalize, transform and innovate the pattern of traditional textile is a hot topic in current research. For the pattern of ethnic home-textiles, the meaning contained is the most important ethnical character. It's the crucial issue that how to preserve and regenerate them in the process of digitization. Herein, combined with the concept of pattern bases, the pattern which was collected is digitalized by CorelDraw or Adobe illustrator software firstly, breaking into basic units afterward, according to the structure of a single pattern through the following three rules.

a. Direct extraction, which is suitable for the single pattern which is symmetrical and all the basic unit is the same. As shown in figure 1.

b. Symmetrical extraction, that is suitable for the vertically, or horizontally symmetrical patterns. One of the symmetrical parts can be selected and break up into basic unit after multi-level pattern bases were extracted. As shown in figure 2.

c. Compositional extraction, which is suitable for some more complicated patterns. Though almost all of the Yormac pattern are symmetrical, every half or quarter part of some patterns do not offer independent information. It is necessary to segment the structure and extract feature units from the composition by taking advantage of the integrity of patterns. As shown in figure 3.

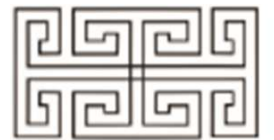

(a)

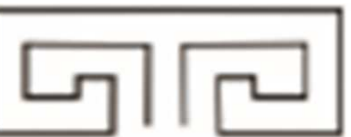

(b)

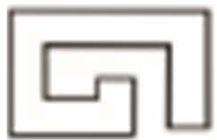

(c)

Figure 1. Direct extraction. 


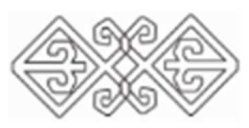

(a)

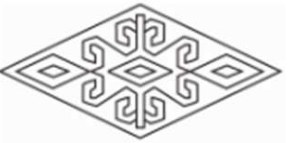

(a)

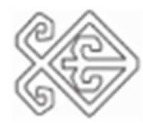

(a-1)

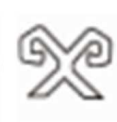

Figure 2. Symmetrical extraction.

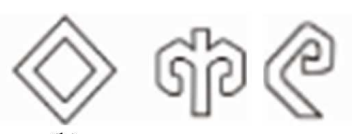

(b)

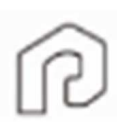

(c)

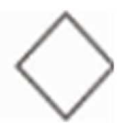

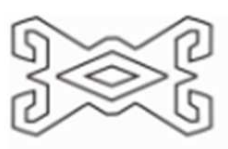

(b)

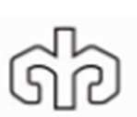

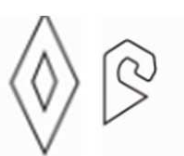

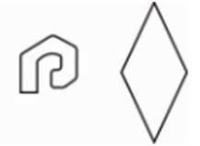

(c)

Figure 3. Compositional extraction.

In figures $1-3$, (a) is original pattern; (a-1) symmetrical extraction; (b) basic pattern of 2th level; and (c) basic pattern of 1 st level, is also called primitive pattern or basic unit of pattern. What is more, the color of Yormac pattern is captured by photoshop (PS), and a Kirgiz style of color-matching scheme is obtained. Finally, a pattern primitive library is constructed.

\subsection{Yormac Pattern Primitive Library}

\subsubsection{Pattern Primitive}

Pattern primitive is basic units of pattern showing certain national characteristics according to Zhao Hai-ying's definition which is derive from metadata. Within a relatively independent information and special meaning in a certain ethnic cultural environment, the feature and characteristics of which are relatively typical, stable, and inheritable. In the process of constructing Kirgiz Yormac pattern primitive library, how to keep and retain the ethnical characteristics of a pattern and make it be independent, stable, recognizable is the key problem. The three rules proposed above are mainly according to the frequency in all of the pattern collected and the imply meaning of the pattern itself.

\subsubsection{Advantages of Construction the Primitive Library}

The significance of constructing Kirgiz Yormac primitive library of pattern is as follows:

First of all, the basic digital information of the patterns can be saved preventing some patterns with special implications from dying away.

Secondly, basic units of pattern which contain independent ethnic identifications and features can be obtained through a multi-level primitive library. And a digital method is established to preserve Kirgiz traditional intangible heritage features.

Furthermore, a novel pattern can be designed immediately through the library. For instance, artistic patterns with Kirgiz aesthetic characteristics can be acquired by copying a basic element in primitive library into a classic structure. For designers, regenerated and design a new pattern with Kirgiz style in short time can be realized based on the library.

The detailed technical route is shown in figure 4 .

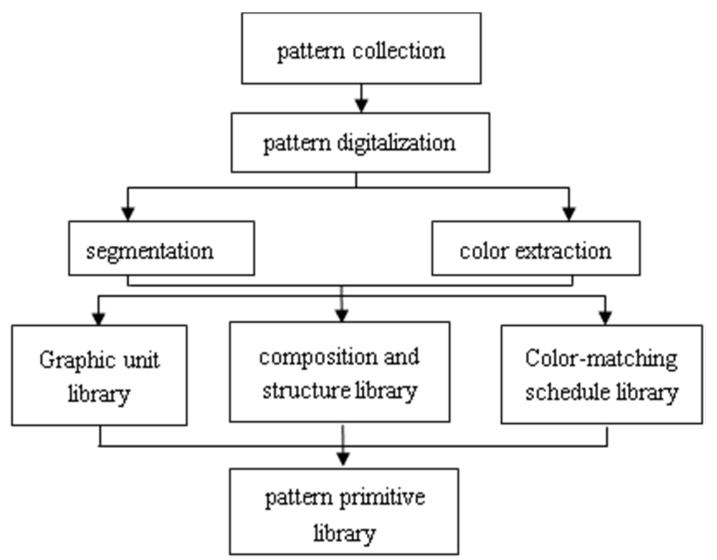

Figure 4. The technical route.

\section{Result and Discussion}

\subsection{Typical Pattern Units}

Classic samples which were collected from three districts (Atux, Aktaw, and Bulunkou) of Kirgiz autonomous prefecture and Ili Kazak autonomous prefecture are sorted base on the pattern, color and region. Three main composition form in Yormac pattern was found through deeply field investigation shown in figure 5. Figure (a) shows a separate discontinuous form, while (b) shows a continuous and suitable structure of pattern. Symmetry is the most remarkable feature of all of the pattern, and that is what extraction rules above according to.

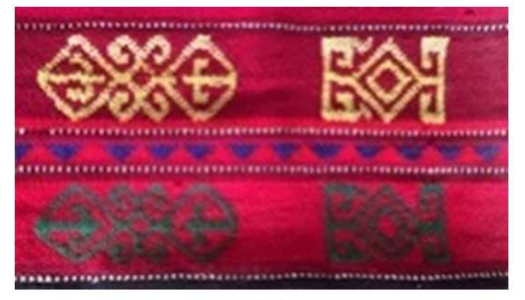

(a)

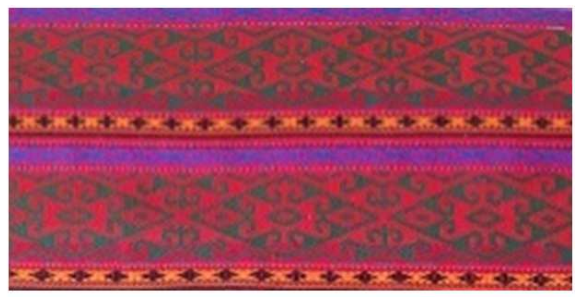

(b)

Figure 5. Construction structure. (the pattern is collected from ATUX district). 
More than 75 figures were statistically analyzed, and all of the different patterns were divided into independent units according to its structure and implications. Among the 142 first-level units obtained, the highest frequency and most representative first-grade typical units and its deformations were classified into 5 categories, as shown in table 1.

Table 1. Typical level-1 units.

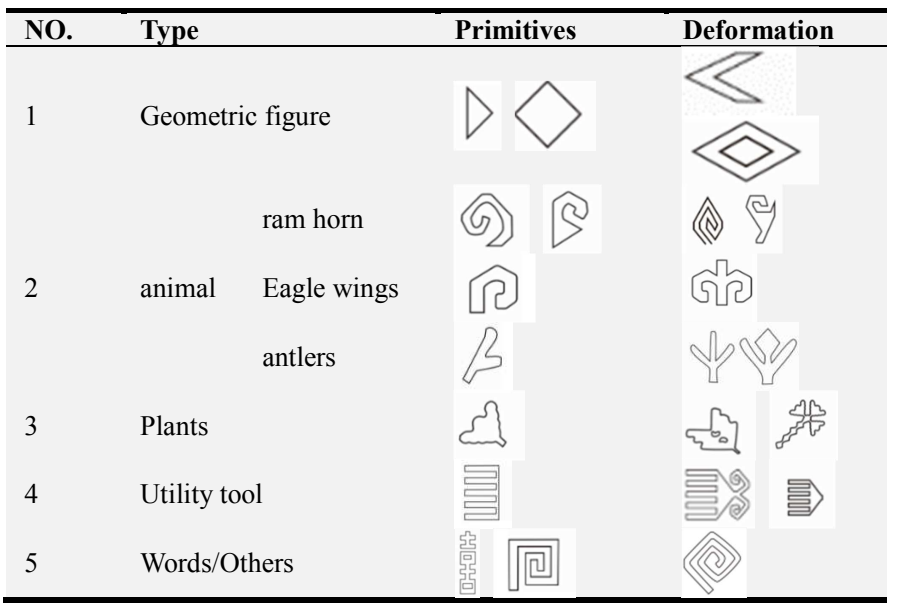

\subsubsection{Geometric Figure}

According to statistics, geometric figure in table 1 contributes $42 \%$ of all of the pattern units divided. In other words, there are $80 \%$ patterns in which was collected that contain geometric units. The basic unit of triangle and diamond is the most prevalent in all of the district visited, and account for almost $90 \%$ of 1 st category. They are often used as the basic unit of a single pattern, or combing with other elements, or used as bands at the interval connecting two different strips. There are other geometric bases such as rectangle and polygon found in the pattern collected from Bulunkou town.

There must be two reasons that could explain here. Firstly, as we all know that regular geometric pattern is easy to manually weave, especially for Kirgiz people who are extremely lack of advanced apparatus. Secondly, geometric patterns play an important role in the history of Chinese decorative pattern. As a part of Chinese ethnic minorities, the culture of Kirgiz is strongly influenced by Chinese traditional artistic culture. With exchanging and communicating culture in a long range, it became a special way for Kirgiz to express the word. According to literature, the implications of triangle and diamond in Kirgiz pattern are continuous mountain and land where Kirgiz survived. With tough surroundings and living conditions, a series delicate textiles and pattern are made. Therefore, the units of triangle and diamond which show Kirgiz living environment and spirit should be treated as typical Kirgiz pattern units and reserved in the pattern primitive library.

\subsubsection{Animal Image and the Words Figure}

The second and fifth categories account for $40 \%$ and $48 \%$ within all of the pattern collected according to the statics, far less than the first class. The basic unit of ram horn which accounts for $71 \%$ mostly in the second category becomes the second common-used figure unit with multiple deformations. In Kirgiz routine life, it is not hard to find that sheep, horses, and yaks which are regarded as living materials, are very common herd. According to literature, ram horn, as a symbol of strength, is deeply loved by Kirgiz people, and widely used as Kirgiz motif. This is in accordance with the field survey as well as the statics. Thus, sheep horn can be regarded as the most representative unit of Kirgiz pattern with independent ethnical information.

Although the figure of eagle wing and antler only account for $12 \%$ among all of the patterns, they are still regarded as indispensable pattern units in the primitive library because of the connotations. It is reported that Kirgiz people is used to raising and training eagles. The flying eagle as a symbol of strength and freedom is regarded as their friends and partners. At the meantime, deer meaning good luck and happiness in Chinese history is also been keen on by Kirgiz people. They turned this kind of love for animals, adore of power and faith for totem into abstract images and weave them into Yormac. Therefore, the flying eagle and antler should be a classical unit of Kirgiz pattern. However, the primitive of eagle wing is mainly found in countryside of Atux city, while the image of antler is obtained from Aktaw. It can be concluded that there are obvious regional differences in pattern using.

Meanwhile, the unit of word “回” is more popular than others in the fifth category. The proportion of the word “回”equals the proportion of triangle unit among all of patterns, proving the importance of the "hui" primitive. In fact, the pattern of “回” is also regard as geometric pattern in Chinese pattern history, and had been long-term inheritance and evolution. It also can be concluded that the pattern in Kirgiz comes down in a continuous line from Chinese pattern history.

Although the 3rd Class and the 4th class together account for $10 \%$ in the pattern units, they are still very important and representative in all of the Yormac pattern. Because that the pattern of comb teeth in category-4 is highly consistent with beating-up tool that has been in Chinese textile history, while the figure of plant, such as the image of flower and leaves are all around the surroundings where Kirgiz live on, both of them has the symbolic implications.

\subsection{The Theme and Structure of Yormac Pattern}

\subsubsection{The Theme Yormac Pattern}

It is found that the theme of Yormac pattern is closely relevant to Kirgiz living-surroundings and daily life, since it can be calculated that almost $88 \%$ patterns collected are structured the pattern units in category -1 and category- 3 , and $41 \%$ contain the unit of animal image in category- 2 . That can also be explained from the life style and habit of Kirgiz. The area with abundant water and grass is suitable for Kirgiz people to graze and live, so they have to migrate with land and season as well as weaving and knitting. The landscape where they come and live becomes the picture and image on their 
belongings naturally. Thus, we can see continuous mountain are conveyed by successive triangles, land is expressed by successive diamond units, and water ripples is delivered by sequential tortuous half horn unit which seems like the deformation ram horn unit in category-2.

The theme of ram horn, flying eagle and antlers is also derived from their life. The adoration of strength against nature, and pursuing of happiness life is always what Kirgiz people are trying to express. Besides, there are some images (not included in table 1) symbolizing the eyes and eyebrows of a girl, which appears in narrower ribbon continuously showing Kirgiz people's yearning for beautiful things.

The themes of Yormac pattern from different area have a little bit different. For instance, geometric pattern and sheep horn images account for almost $74 \%$ in the patterns collected at Aktaw, while account for $60 \%$ and $41 \%$ in the patterns collected at Bulunkou and Atux. There are no plants and flowers that were figured out in Bulunkou and Aktao, but these can be seen in Atux region. Meanwhile, there are also figures like "xi"(acquired at Atux) and "hui" (gain from Bulunkou town mostly) which are highly in harmony with Chinese traditional culture, also can fully reflect the consensus of artistic aesthetic in the long-time communication between Kirgiz and Han nationality.

\subsubsection{The Composition and Structure}

Symmetry and balance are particularly outstanding features in Yormac pattern. Due to the size of the loom, the width of
Yormac fabric is limited. Thus, single pattern, two-side continuous arrangement is typical structure of pattern in Yormac. Continuous pattern grouped by individual figures arrayed according to a certain rule, or a series same figure by changing the direction and arrangement, making the entire pattern full of sense of rhythm. There are also symmetrical, vertical, wavy, scattered and oblique arrangements in continuous pattern, which is highly consistent with Chinese traditional ideology, that are simple but complex, balance but changeable, scatter but integral characters.

When several strips of Yormac are sewn together forming a whole fabric, the organization and composition of patterns are changing. The symmetrical aesthetic principles in individual and continuous patterns are fully deductive, showing a high consistency between Kirgiz culture and traditional Chinese culture.

\subsection{The Color of Yormac Pattern}

The color expression of national decorative patterns can show the color preference of a nation. Many literatures showed the custom of using and collocating color, and analyzed the relationship between Kirgiz color preference and the spiritual belief. Through statistical analysis of the color in the collected sample, it is found that the frequently-used colors of the products mainly include red, green, yellow, blue, black and white. Table 2 is shown the proportion of various colors used between Aktaw and Atux region.

Table 2. Proportion of popular color of Yormac.

\begin{tabular}{|c|c|c|c|c|c|c|}
\hline region & Red & Green & Yellow & Blue & Black & White \\
\hline Aktaw & $33.8 \%$ & $15 \%$ & $20 \%$ & $11.3 \%$ & $16.3 \%$ & $2.5 \%$ \\
\hline Atux & $38.2 \%$ & $17.1 \%$ & $18.4 \%$ & $7.9 \%$ & $7.9 \%$ & $6.5 \%$ \\
\hline
\end{tabular}

It can be seen from the table 2 that red, green, yellow and blue account for more than $80 \%$ in all collected samples. Red is the color with the highest frequency in two regions, accounting for $72 \%$ of the total products collected, becoming the most popular color among Kirgiz people. Some scholars believe that red is the symbol of enthusiasm and unrestrained which is relevant to Kirgiz hospitality, lively and cheerful character, while some other scholars believe that red is the symbol of fire, and related to shamanism in Kirgiz long religious history. Red is widely used as the background color of Yormac, decorating with green, blue, yellow or black figures, forming inimitable artistic color style. Yellow (include Orange, lemon yellow) is less popular than red, accounting for more than $40 \%$ of the total products collected. Orange is used as the background color, matched with black, blue and green patterns, forming a warm style. Lemon yellow often serves as decorative pattern color, matched with red, blue, and black, gaining a peaceful style. The color of green, blue, black, and white is often used as pattern colors, strongly contrasting with the purer red background. Additionally, a small amount of yellow- purple patterns are seen at Atux. And there is also some rare color match schedule at Aktaw, such as black background matched with a orange pattern, dark brown background matched with a light green pattern, etc. Black account for more proportions in Aktaw than Atux, which is usually in combination with red, blue and orange.

As well as the patterns, the color of Yormac comes from Kirgiz living surroundings and is in harmony with the high saturation of the natural color, such as the warm color of the sun, the green of spring and summer, the white of snow mountains, and the yellow of autumn. In the schedule of color matching, warm colors such as red and yellow are given priority to, and the patterns are often matched with contrasting and complementary colors, which form a warm and lively style. When decorated in a yurt, a warm and gorgeous feeling is presented. The warm color-style also can complement the cold environment and surroundings. According to field investigation, it is revealed that the older Yormac is made of wool, camel hair and other animal hair, which is dyed by hand with natural or chemical dyestuff. The saturation and fastness of color are not as good as current imitations made of acrylic. Acrylic Yormac with brighter color compared with woolen Yormac, lower price, the twisting process simplifying and other advantages appealed by Kirgiz people nowadays, and gradually replace the market. 
Only in some traditional Kirgiz home can we find the kind of fabric made of pure wool.

\section{Conclusions}

The theme, color, structure of Kirgiz Yormac pattern is driving from life and used for life, expressing the enthusiasm of life, yearning for happiness, and the spirit of hard working of Kirgiz tribe. The custom, implication, spirit of Kirgiz shown in patterns come down in one continuous line with national pattern, and highly consistent with the culture and tradition of Chinese nation. Therefore, Kirgiz Yormac with its fruitful products and unique patterns turns into the typical representative of Kirgiz intangible cultural heritage. By constructing multi-level pattern primitive library though decomposing and segmenting pattern units which obtain the characteristics of the Kirgiz ethnic style, pattern primitives library including graphic unit, composition and structure, and color-matching library not only saved typical pattern with the Kirgiz intangible cultural heritage characteristics, but also simplified redesigning process. Thus, this work done in this paper lays the foundation for better protection, inheritance and innovation of Kirgiz motif culture.

\section{Funding}

This work is supported by Ministry of Education in China, Humanities and Social Sciences Research Youth Foundation (No.18XJJC850001).

\section{References}

[1] Y. Miaomiao, "The Art Form of Kirgiz Ethnic Decoration Pattern in Xinjiang," J. Art and Design|, vol. 8, pp. 104-106, 2016.

[2] W. Xueyu, C. Meng, "Distribution and characteristics of Kirgiz population change in China," J. Northwest population, vol. 4, pp. 55-61, 2003.

[3] Y. Wenqi, G. Mengjia, X. Aimin, et al., "An analysis on weaving techniques and garment structures of the ground loom of the Kirgiz in Xinjiang," J. Journal of Silk, vol., pp. 1-9, Jul. 2019.

[4] Q. Juan, X. Aimin, X. Xiaoyu, et al., "Study on the Weaving Process of Kirgiz Traditional Woolen Fabric," J. Chinese Journal of Design, vol. 12, pp. 116-117, 2017.

[5] Q. Juan, X. Aimin, R. Lei, et al., "Research on inheritance and innovation of the Kirgiz's camel fabric Qiepukan," J. Wool Textile Journal, vol. 46, pp. 72-75, Aug. 2018.
[6] Z. Chuiti, "A Primary research of the Beauty of Folk Batik Patterns in Guizhou," J. Guizhou Literature and History Series, vol., pp. 148-152, 1991.

[7] T. Shaoxu, "A preliminary study on the brocade patterns of Tujia," J. Journal of the Central University for Nationalities, vol., pp. 90-93, 1989.

[8] Z. Yaozhen, "Uygur folk dyeing and embroidery patterns," J. Chinese Journal of Design, vol., pp. 14-15, 1986.

[9] X. Zhongiie, "Traditional patterns and colors of Nanjing Brocade," J. Journal of Nanjing University of the Arts (Music and Performance Edition), vol., pp. 125-132, 1980.

[10] W. Xueyu, "The Traditional Kirgiz Needlecraft in the Transformation Period," J. Journal of Xinjiang University (Philosophy, Humanities \& Social Science), vol. 42, pp. 72-76, Sep. 2014.

[11] W. Xueyu, "The Females and the Traditional Culture of the Kirgiz Minority Group," J. Journal of KashgarTeachers College, vol. 33, pp. 40-43, 2013.

[12] W. Xueyu, "Analysis and Exploration of Kirgiz Tapestry," J. journal of Xinjiang arts University, vol. 7, pp. 7-11, Sep. 2014.

[13] W. Xueyu, "On Kirgiz Traditional Craftsmanship during the Social Transformation Period," J. Journal of Guizhou Normal University (Social Science), vol. 201, pp. 83-90, 2016.

[14] L. Jianguo, "An Analysis of Khalkhas Design Pattern," J. Chinese Journal of Design, vol. 173, pp. 89-90, 2007.

[15] Y. Miaomiao, "Cultural analysis of Kirgiz national decorative pattern art in China," J. Textile Dyeing and Finishing Journal, vol. 40, pp. $48-51,2018$

[16] C. Jiazhou, H. Wenwen, M. Yongwei, et al., "Pattern Analysis and Digitization Modeling of Papercutting Textures," J. Journal of Computer-Aided Design \& Computer Graphics, vol. 28, pp. 1465-1474, 2016.

[17] Z. Haiying, Z. Junhui, "Xinjiang Folk Art Patterns Digital Technology," J. Joournal of computer system application, vol. 20, pp. 167-172, 2011.

[18] Z. Haiying, H. Wen, X. Guang-Mei, "A New Method for Pattern Generation," J. Joournal of computer system application, vol. 20, pp. 85-89+97, 2011.

[19] Z. Haiying, X. Zhengguang, Z. Junhui, "Research on Uygur carpet pattern digital technology," J. Journal of Textile Research, vol. 33, pp. 142-145, 2012.

[20] W. Yun, "The Digital Construction of the Basic Elements of Xinjiang Ethnic Costumes and Applied to the Design," J. Chinese Journal of Design, vol. 2, pp. 85-87, 2017. 